\title{
Comparing the Functionality between Virtual Reality and Mixed Reality for Architecture and Construction Uses
}

\author{
Yilei Huang, Samjhana Shakya and Temitope Odeleye \\ Department of Construction \& Operations Management, South Dakota State University, Brookings, SD 57007, USA
}

\begin{abstract}
Virtual Reality (VR) and Mixed Reality (MR) offer unique opportunities for the architecture and construction industry through different approaches with building information modeling (BIM). While VR offers architecture and construction practitioners the ability to personally experience the built environment in an immersive, MR with its unique ability of overlaying digital information in the real world allows practitioners to perform on-site visualization for construction planning and as-built verification. With their similar but distinct characteristics, VR and MR offer a variety of functionality to the architecture and construction industry that often confuses practitioners on what to choose to best fit their needs. To clarify this confusion, this paper investigates the available technologies of VR and MR in terms of both hardware and software and compares the functionality between the two for architecture and construction uses. While VR hardware has been developed into three categories based on their connection types and tracking methods, MR hardware has mainly focused on standalone devices. Eight VR software and nine MR software have been identified, investigated, and compared. This paper provides the latest information for architecture and construction practitioners on how VR and MR hardware and software work similarly and differently.
\end{abstract}

Key words: Virtual reality, mixed reality, functionality, construction, comparison.

\section{Introduction}

With the increasing popularity of virtual reality (VR) and mixed reality (MR) in the consumer market, the architecture and construction industry has quickly recognized their value in visualizing designs and implementing on-site analyses with building information modeling (BIM). While VR offers architecture and construction practitioners the ability to personally experience the built environment in an immersive manner that no other existing visualization tools can ever match, MR with its unique ability of overlaying digital information in the real world allows practitioners to perform on-site visualization for construction planning and as-built verification.

With their similar but distinct characteristics, VR and MR offer a variety of functionality to the architecture and construction industry that often

Corresponding author: Yilei Huang, Ph.D., CM-BIM, assistant professor, research fields: building information modeling, virtual reality and mixed reality in construction management. confuses practitioners on what to choose to best fit their needs. To clarify this confusion, this paper investigates the available technologies of VR and MR in terms of both hardware and software and compares the functionality between the two for architecture and construction uses.

\section{Background}

The technology of VR and MR has developed into a stage that has divided the hardware into three categories based on their connection type, as summarized in Table 1. In the PC-based connection type, the hardware is required to be connected to a capable personal computer (PC) through cables. First generation VR devices, including Oculus Rift and HTC Vive as well as newer and high-end VR devices, such as HTC Vive Pro, Pimax 5K/8K, and Valve Index, also require separation base stations for tracking, while second generation VR devices use inside-out tracking approaches through embedded cameras to eliminate separate base stations, such as 
Table 1 A summary of existing virtual reality and mixed reality headsets as of 2019.

\begin{tabular}{|c|c|c|c|c|}
\hline \multicolumn{3}{|c|}{ Headset } & \multirow{2}{*}{ Virtual reality } & \multirow{2}{*}{ Mixed reality } \\
\hline Connection & Tracking & & & \\
\hline \multirow[t]{2}{*}{ PC-based } & \multirow{2}{*}{$\begin{array}{l}6 \mathrm{DOF} \\
\text { (position + rotation) }\end{array}$} & Base stations & $\begin{array}{l}\text { Oculus Rift } \\
\text { HTC Vive/Pro/Eye } \\
\text { Pimax 5K/8K } \\
\text { Valve Index } \\
\end{array}$ & \\
\hline & & Inside-out & $\begin{array}{l}\text { Oculus Rift S } \\
\text { HTC Vive Cosmos } \\
\text { WMR VR headsets }\end{array}$ & Meta 2 \\
\hline \multirow[t]{2}{*}{ Standalone } & $\begin{array}{l}6 \mathrm{DOF} \\
\text { (position + rotation) }\end{array}$ & Inside-out & $\begin{array}{l}\text { Oculus Quest } \\
\text { HTC Vive Focus/Plus } \\
\text { Lenovo Mirage Solo } \\
\end{array}$ & $\begin{array}{l}\text { Microsoft HoloLens } 1 \& 2 \\
\text { Magic Leap One } \\
\text { Lenovo ThinkReality A6 }\end{array}$ \\
\hline & 3 DOF (rotation) & & Oculus Go & \\
\hline Cellphone-based & 3 DOF (rotation) & & $\begin{array}{l}\text { Samsung Gear VR } \\
\text { Google Daydream View } \\
\text { Generic VR headsets }\end{array}$ & \\
\hline
\end{tabular}

Oculus Rift S, HTC Vive Cosmos, and a variety of Windows Mixed Reality VR headsets. All PC-based VR devices support six degrees of freedom (DOF) of tracking, which includes movement along and rotation around the three perpendicular axes. Meta 2, as the only PC-based MR device, has been phased out and discontinued.

Standalone devices have been the main development trend due to their convenience and portability. Except for Oculus Go, all VR and MR devices use the inside-out approach to support 6 DOF tracking, including newer VR devices such as Oculus Quest, HTC Vive Focus, and Lenovo Mirage Solo, as well as all MR devices including Microsoft HoloLens 1 and 2, Magic Leap One, and Lenovo ThinkReality A6. Oculus Go, as a lower-end VR device, is not equipped with any embedded cameras and thus can only perform 3 DOF tracking, or rotation only. Cellphone-based VR devices, as the entry level VR category, are merely as a housing of VR lenses for capable cellphones. Much depended on the cellphone itself, these VR devices work similarly to Oculus Go and only support 3 DOF tracking.

\section{Literature Review}

Due to the unique benefits that VR brings to the industry, research efforts have started to investigate its uses in various areas in architecture and construction.
Froehlich and Azhar [1] evaluated the use of Oculus Rift in construction safety training and jobsite management, while Petrova et al. [2] evaluated such use in end-user involvement in building design. Dayan and Sasks [3] investigated the enhancement of cognition using Oculus Rift in apartment customization. Ozcelik et al. [4] and Carneiro and Becerik-Gerber [5] studied the use of Oculus Rift in understanding occupant-system interactions related to thermal changes and lighting quality, respectively. Soman and Whyte [6] and Lovreglio et al. [7] developed a framework with VR visualization for real-time construction progress monitoring and earthquake evacuation, respectively. Asgari and Rahimian [8] investigated different VR tracking devices for construction process optimization and defect prevention.

Early research efforts have also been devoted to implementing MR and HoloLens in architectural and industrial design and construction. Alsafouri and Ayer [9] first designed a methodology for generating marker-based MR environments for various mobile computing devices, such as smartphones and tablets, to enable design and constructability review using existing BIM contents. Chalhoub and Ayer [10] then investigated the perception of field workers in using a BIM model through HoloLens as the construction documents to assemble electrical conduits compared 
with traditional paper-based communication in 2D drawings. Chalhoub and Ayer [11] further examined its impact on construction performance in terms of productivity and quality based on the same electrical conduit assembly, and discovered that using BIM models through MR significantly increased productivity rate, lowered the number of installation errors, and reduced the time needed to understand the design during the electrical conduit assembly process.

\section{Methodology}

Through a comprehensive literature review and an internet search, a list of existing VR and MR software has been identified, as summarized in Table 2, including their developer, application name, and the purpose of VR or MR use. Eight VR applications have been identified, among which seven are developed for BIM uses, including Revit Live, Enscape, Fuzor, Revizto, InsiteVR, Prospect, and Kubity, while Composer is developed for 3D design in various fields. Nine MR applications have been identified, among which 3D Viewer Beta is developed for general 3D object viewing, Vyzn and Prism are specifically purposed for MR presentations, and the rest seven are developed for BIM uses, including SketchUp Viewer, Trimble Connect, Fuzor AR, BIM Holoview, MR Builder, and HoloLive 3D. The functionality of each VR and MR application was investigated and compared, and the results are presented as follows in terms of their common and unique features.

\section{Results}

\subsection{Common Functionality}

\subsubsection{Compatibility}

Most VR and MR software support the popular 3D and BIM file formats, including Revit, SketchUp, and fbx files. While Revit and SketchUp files are commonly supported by VR software due to the convenience of VR plugins in Revit and SketchUp, fbx files are better supported by MR software as a typical 3D format for conversion. In addition, some other 3D formats are also supported by a few VR software, such as Rhino, 3ds Max, Navisworks, and ArchiCAD.

\subsubsection{Miniature}

In the miniature viewing mode or tabletop mode, most VR software allows the model to be rotated and scaled with controllers, while most MR software also supports moving the miniature model and anchoring it to a fixed position in the physical space. The combination of moving, rotating, and scaling tools provides the freedom of viewing the miniature model at any size, angle, and place.

\subsubsection{Utilities}

Most VR and MR software share some common utility tools for architecture and construction uses, including measurement, annotation, layer control, and object information. The measurement tool allows the user to measure the distance either between two points or the floor to ceiling height in VR software, and also

Table 2 A summary of existing virtual reality and mixed reality software as of 2019.

\begin{tabular}{|c|c|c|c|c|c|}
\hline \multicolumn{3}{|c|}{ Virtual reality software } & \multicolumn{3}{|c|}{ Mixed reality software } \\
\hline Developer & Application & Purpose & Developer & Application & Purpose \\
\hline SimLab & Composer & $3 \mathrm{D}$ & Microsoft & 3D Viewer Beta & $3 \mathrm{D}$ \\
\hline Autodesk & Revit Live & BIM & Trimble & SketchUp Viewer & BIM \\
\hline Enscape & Enscape & BIM & Trimble & Trimble Connect & BIM \\
\hline Kalloc & Fuzor & BIM & Kalloc & Fuzor AR & BIM \\
\hline Vizerra & Revizto & BIM & BIM Holoview & BIM Holoview & BIM \\
\hline Vrban & InsiteVR & BIM & Holo Group & MR Builder & BIM \\
\hline IrisVR & Prospect & BIM & VisualLive & HoloLive 3D & BIM \\
\hline \multirow[t]{2}{*}{ Kubity } & Kubity & BIM & Zengalt & Vyzn & Presentation \\
\hline & & & Object Theory & Prism & Presentation \\
\hline
\end{tabular}


enables distance measurement in the physical space or between the model and physical space in MR software. Annotation tools include markups and snapshots on the model that provides feedback to other BIM users while they inspect the model with the VR or MR software. Layer control enables turning individual BIM component types on and off for a better viewing experience providing they are separated in their BIM authoring software. Object information allows the user to inspect the properties of the selected BIM components when they are created in the authoring software.

\subsection{Unique Functionality}

\subsubsection{Full Scale}

The functionality tools at full scale only apply in MR software since the model needs to be overlaid in a physical space, as summarized in Table 3. SketchUp Viewer and Trimble Connect are the only two applications that support full scale size adjustment, which allows the application to slightly adjust the size of all models to ensure an exact match of size at full scale. HoloLens tends to display 3D models slightly smaller than their true size by about $1-2 \%$. This discrepancy is negligible for small objects, but for BIM models the difference can go up to a few inches. The full scale adjustment feature allows SketchUp Viewer and Trimble Connect to compensate this discrepancy from HoloLens when overlaying BIM models on-site at full scale. Automatic alignment allows BIM models to be automatically placed at the correct position on-site at full scale. These models contain walls or floors that are either existing or have already been installed as reference planes to align with the physical walls or floors at full scale. Once the size, position, and direction of the walls or floors in the BIM model match those at the physical site, all other objects in the model will then indicate the correct positions of on-site installation, which tremendously facilitates pre-construction planning and as-built verification. The user can then walk freely to inspect the model against the physical space while the model stays stationary. Trimble Connect, BIM Holoview, MR Builder, HoloLive 3D, and Fuzor AR support automatic alignment through different methods.

\subsubsection{Simulation}

Simulation tools only apply in VR software since the PC-based VR devices specialize in 3D image rendering by taking the advantage of the connected PC, as summarized in Table 4. The VR scenes rendered by Revit Live, Enscape, Fuzor, and Composer look more realistic than the other applications due to the rendering engine and materials used, and consequently demand more computer resources. The same four applications also simulate dynamic object effects, which allow the user to observe the natural movement of certain objects in the VR scene, such as burning flames, water waves and reflections, smoke, swinging leaves, spinning fans, television contents, etc. These dynamic effects consume

Table 3 Mixed reality software full scale tools.

\begin{tabular}{lll}
\hline Application & \multicolumn{2}{c}{ Mixed reality software } \\
\hline 3D Viewer Beta & Full scale tools \\
SketchUp Viewer & Size adjustment & \\
Trimble Connect & Size adjustment & Automatic alignment \\
Fuzor AR & & Automatic alignment \\
BIM Holoview & & Automatic alignment \\
MR Builder & Automatic alignment \\
HoloLive 3D & Automatic alignment \\
Vyzn & \\
Prism & \\
\hline
\end{tabular}


Table 4 Virtual reality software simulation tools.

\begin{tabular}{|c|c|c|c|c|}
\hline \multicolumn{5}{|c|}{ Virtual reality software } \\
\hline \multirow{2}{*}{$\frac{\text { Application }}{\text { Composer }}$} & \multicolumn{4}{|c|}{ Simulation tools } \\
\hline & Rendering & Object & Daylight & Lighting \\
\hline Revit Live & Rendering & Object & Daylight & Lighting \\
\hline Enscape & Rendering & Object & Daylight & Lighting \\
\hline Fuzor & Rendering & Object & Daylight & Lighting \\
\hline Revizto & & & Daylight & Lighting \\
\hline InsiteVR & & & Daylight & Lighting \\
\hline Prospect & & & Daylight & Lighting \\
\hline Kubity & & & Daylight & Lighting \\
\hline
\end{tabular}

Table 5 VR and MR software collaboration features.

\begin{tabular}{|c|c|c|c|c|}
\hline \multicolumn{2}{|c|}{ Virtual reality } & \multicolumn{3}{|c|}{ Mixed reality } \\
\hline Application & Collaboration & Application & & Collaboration \\
\hline Composer & & 3D Viewer & & \\
\hline Revit Live & & SketchUp & Sharing & Co-location \\
\hline Enscape & & TrimbleConnect & Sharing & \\
\hline Fuzor & Sharing & Fuzor AR & Sharing & Co-location \\
\hline Revizto & Sharing & BIM Holoview & & \\
\hline InsiteVR & Sharing & MR Builder & & \\
\hline Prospect & Sharing & HoloLive 3D & & \\
\hline \multirow[t]{2}{*}{ Kubity } & & Vyzn & Sharing & Co-location \\
\hline & & Prism & Sharing & \\
\hline
\end{tabular}

much higher computer resources since they are constantly changing. Daylight simulation allows the VR user to change the current time in a day to adjust the sun position and observe the effects of daylight change in the built environment in the VR scene. Lighting simulation allows the user to observe and adjust the lighting effects in the VR scene when artificial lighting is added in the BIM model. Lighting effects can be observed when the time of day is set to night and artificial lighting becomes the main lighting source. All VR software supports both daylight and lighting simulation.

\subsubsection{Collaboration}

Both VR and MR software support collaboration with various approaches, as summarized in Table 5 . The collaboration feature allows multiple VR or MR users, each wearing his/her own headset, to view the same built environment at the same time. All participants can walk freely in the shared virtual environment and observe the presence of one another in the virtual scene. A headset or avatar model with the user's name is displayed to represent the positions of each participant in the scene. In addition, participants can present a laser dot from their avatar models with the controllers to guide the project team on the same building component during a team discussion. MR software, including SketchUp Viewer, Fuzor AR, and Vyzn, additionally supports co-location, which also shares the location of each HoloLens in the session. Every user needs to identify a common point in the physical space to allow the model to be placed at the same position and direction for all participants. Each participant will then be represented by an avatar to indicate their position in the collaboration session and present their focus point with a laser beam. Non-co-located collaboration, however, is not able to accurately represent each participant's actual position in the session.

\section{Conclusions}

The architecture and construction industry has quickly recognized the value of VR and MR in 
visualizing designs and implementing on-site analyses with BIM due to their increasing popularity in the consumer market. While VR offers architecture and construction practitioners the ability to personally experience the built environment in an immersive manner that no other existing visualization tools can ever match, MR with its unique ability of overlaying digital information in the real world allows practitioners to perform on-site visualization for construction planning and as-built verification. With their similar but distinct characteristics, VR and MR offer a variety of functionality to the architecture and construction industry that often confuses practitioners on what to choose to best fit their needs.

To clarify this confusion, this paper investigates the available technologies of VR and MR in terms of both hardware and software and compares the functionality between the two for architecture and construction uses. While VR hardware has been developed into three categories based on their connection types and tracking methods, MR hardware has mainly focused on standalone devices. Eight VR software and nine MR software have been identified, investigated, and compared. While some of the common functionality includes file type compatibility, miniature viewing mode, and utility tools, there are unique functionality features for both VR and MR software, including full scale tools only for MR, simulation tools only for VR, and collaboration approaches that differ between VR and MR software. This paper provides the latest information for architecture and construction practitioners on how VR and MR hardware and software work similarly and differently.

This study was partially sponsored by the Thomas Glavinich ELECTRI International 2018 Early Career Award. The authors would like to thank ELECTRI International and Thompson Electric Company for their support.

\section{References}

[1] Froehlich, M. A., and Azhar, S. 2016. "Investigating Virtual Reality Headset Applications in Construction.” In
Proceedings of the 52nd Associated Schools of Construction Annual International Conference, Provo, UT.

[2] Petrova, E., Rasmussen, M. B., Jensen, R. L., and Svidt, K. 2017. "Integrating Virtual Reality and BIM for End-User Involvement in Design: A Case Study.” In Proceedings of the Joint Conference on Computing in Construction (JC3), Heraklion, Greece, 699-706.

[3] Dayan, A., and Sacks, R. 2017. "Cognition Enhancement Using Virtual Reality in Apartment Customization.” In Proceedings of the Joint Conference on Computing in Construction (JC3), Heraklion, Greece, 571-8.

[4] Ozcelik, G., Becerik-Gerber, B., Ghahramani, A., and Wang, Y. 2017. "Can Immersive Virtual Environments Be Used for Understanding Occupant-System Interactions Under Thermal Stimuli?” In Proceedings of the Joint Conference on Computing in Construction (JC3), Heraklion, Greece, 357-64.

[5] Carneiro, J. P., and Becerik-Gerber, B. 2017. "Impact of Immersive and Interactive Information Visualization on Occupant's Lighting Choices." In Proceedings of the Joint Conference on Computing in Construction (JC3), Heraklion, Greece, 465-73.

[6] Soman, R. K., and Whyte, J. K. 2017. “A Framework for Cloud-Based Virtual and Augmented Reality Using Real-Time Information for Construction Progress Monitoring." In Proceedings of the Joint Conference on Computing in Construction (JC3), Heraklion, Greece, 833-40.

[7] Lovreglio, R., Gonzalez, V., Amor, R., Spearpoint, M., Thomas, J., Trotter, M., and Sacks, R. 2017. "The Need for Enhancing Earthquake Evacuee Safety by Using Virtual Reality Serious Games.” In Proceedings of the Joint Conference on Computing in Construction (JC3), Heraklion, Greece, 381-9.

[8] Asgari, Z., and Rahimian, F. P. 2017. “Advanced Virtual Reality Applications and Intelligent Agents for Construction Process Optimisation and Defect Prevention.” Procedia Engineering 196: 1130-7.

[9] Alsafouri, S., and Ayer, S. K. 2017. “A Step-by-Step Procedure for Implementing Mixed Reality Visualization Interfaces in Design and Constructability Review Sessions." In Proceedings of the Joint Conference on Computing in Construction (JC3), Heraklion, Greece, 913-21.

[10] Chalhoub, J., and Ayer, S. K. 2017. "Perception of Industry Professionals about Mixed Reality for Electrical Prefabrication.” In Proceedings of the 2017 Canadian Society for Civil Engineering Annual Conference, Vancouver, Canada, 83-1-10.

[11] Chalhoub, J., and Ayer, S. K. 2018. "Using Mixed Reality for Electrical Construction Design Communication.” Automation in Construction 86: 1-10. 